\title{
SOME PROPERTIES OF HILBERT SCALES
}

\author{
ROBERT BONIC ${ }^{1}$
}

1. Hilbert scales. Suppose $E_{0}$ is a separable complex Hilbert space with inner product (, ) $)_{0}$. Let $A: E_{0} \rightarrow E_{0}$ be a completely continuous linear mapping with $A>0$. Then $A$ can be represented in the form $A u=\sum_{j=1}^{\infty} \lambda_{j}\left(u, a_{j}\right)_{0} a_{j}$ where $\left\{a_{j}\right\}$ is a complete orthonormal set in $E_{0}, \lambda_{1} \geqq \lambda_{2} \geqq \cdots>0$, and $\lim \lambda_{j}=0$, (see [1, Chapter 1]). Let $E=\bigcap_{n=0}^{\infty} A^{n} E_{0}$ and for $u, v \in E, \alpha \in R$ define $(u, v)_{\alpha}=\left(A^{-\alpha} u, A^{-\alpha} v\right)_{0}$. Denote by $E_{\alpha}, E$ equipped with the \|\|$_{\alpha}=(,)_{\alpha}^{1 / 2}$ topology, and let $E_{\alpha}$ denote the completion. It follows that $E=\bigcap_{\alpha} E_{\alpha}$. The family $\alpha \rightarrow E_{\alpha}$ is called the Hilbert scale defined by $A$, and $E$ is called the center (see [2, p. 93]). We will suppose that $E$ carries the weakest topology in which all inclusions $E \rightarrow E_{\alpha}$ are continuous. It follows that $E$ is a perfect space in the sense of [1] and $\cup_{\alpha} E_{\alpha}=E^{*}$ is its dual space.

Since $\|u\|_{\alpha}=\left\|A^{\beta} u\right\|_{\alpha+\beta}$ for all $u \in E$ and $\alpha, \beta \in R$ it follows that $A^{\beta}$ extends to an isometry from $E_{\alpha}$ to $E_{\alpha+\beta}$, which we will also denote by $A^{\beta}$. Also, it follows from the definition that $\left\{\lambda_{j}^{\alpha} a_{j}\right\}$ is a complete orthonormal set in $E_{\alpha}$ and $\left(u, a_{j}\right)_{\alpha} \lambda_{j}^{2 \alpha}=\left(u, a_{j}\right)_{0}$ for $u \in E_{\alpha}, \alpha \geqq 0$.

An alternative method of introducing Hilbert scales follows from the observation that if $i: E_{1} \rightarrow E_{0}$ denotes the inclusion map, then $A=\left(i^{*} i\right)^{1 / 2}$. Hence, suppose $H_{0}$ and $H_{1}$ are complex Hilbert spaces, $H_{1}$ is dense in $H_{0}$, and the inclusion map $i: H_{1} \rightarrow H_{0}$ is completely continuous. Then $i$ has the form $u=i(u)=\sum_{j=1}^{\infty} \alpha_{j}\left(u, b_{j}\right)_{1} a_{j}$ where $\lim \alpha_{j}=0$, and $\left\{a_{j}\right\}$ and $\left\{b_{j}\right\}$ are bases in $H_{0}$ and $H_{1}$ respectively. It follows that $i^{*} i \in \mathcal{L}\left(H_{1}, H_{1}\right)$ has the form $i^{*} i(u)=\sum\left|\alpha_{i}\right|^{2}\left(u, b_{i}\right)_{1} b_{i}$ which extends to $H_{0}$ by setting $i^{*} i(v)=\sum\left|\alpha_{i}\right|^{2}\left(v, a_{i}\right)_{0} a_{i}$. The pair $\left\{H_{0},\left(i^{*} i\right)^{1 / 2}\right\}$ then defines a Hilbert scale as above.

An important class of Hilbert scales are those whose centers are nuclear spaces. It is not difficult to show that if $\alpha \rightarrow E_{\alpha}$ is a Hilbert scale defined by $A$, then the center is nuclear iff $\sum_{i=1}^{\infty} \lambda_{i}^{\sigma}<\infty$ for some $\sigma>0$.

Two simple examples are given below, and other examples can be found in [2] and [5].

(a) Suppose $E$ denotes the set of all $C^{\infty}$ mappings $u: R \rightarrow C$ having period one, and for $u, v \in E$ define $(u, v)_{0}=\int_{0}^{1} u(t) \bar{v}(t) d t$ and $(u, v)_{1}$ $=(u, v)_{0}+(D u, D v)_{0}$. Let $E_{i}$ denote the completion of $E$ with respect to $(,)_{i}^{1 / 2}$. Then the inclusion map $i: E_{1} \rightarrow E_{0}$ is completely continuous

Received by the editors November 14, 1966.

1 Research supported in part under NSF grant GP-5619. 
and $A=\left(i^{*} i\right)^{1 / 2}$ defines a Hilbert scale with center again $E$. Since $A^{-2}=I-D^{2}$ it follows that $E$ is nuclear.

(b) Suppose $H$ is a separable Hilbert space, $\lambda_{1} \geqq \lambda_{2} \geqq \cdots>0$, and $\lim \lambda_{i}=0$. Let $H_{\alpha}=\left\{a=\left(a_{1}, a_{2}, \cdots\right): a_{i} \in H\right.$ and $\sum\left\|a_{i}\right\|{ }^{2} \lambda_{i}^{-2 \alpha}$ $<\infty\}$, and set $(a, b)_{\alpha}=\sum\left(a_{i}, b_{i}\right) \lambda_{i}^{-2 \alpha}$. Then $\alpha \rightarrow H_{\alpha}$ is a Hilbert scale whose center is nuclear iff $\sum \lambda_{i}^{\sigma}<\infty$ for some $\sigma>0$.

2. Smoothing operators. Suppose $\alpha \rightarrow E_{\alpha}$ is a Hilbert scale defined by $A$, and let $u \in E_{\alpha}$. Then $u=\sum_{i=1}^{\infty}\left(u, \lambda_{i}^{\alpha} a_{i}\right)_{\alpha} \lambda_{i}^{\alpha} a_{i}$. Define $P_{n} u$ $=\sum_{i=1}^{n}\left(u, \lambda_{i}^{\alpha} a_{i}\right)_{\alpha} \lambda_{i}^{\alpha} a_{i}$. It follows that $P_{n}: E^{*} \rightarrow E$ is well defined, $P_{n}^{2}=P_{n}, P_{n} A^{\alpha}=A^{\alpha} P_{n}$ for all $\alpha \in R$; and for $u \in E_{\alpha},\left\|P_{n} u-u\right\|_{\alpha} \rightarrow 0$.

When the center of the scale is nuclear a subsequence $S_{k}=P_{n(k)}$ can be given that is very similar to the smoothing operators used in [4] and [6]. The bounds given below for the norms of these operators are a bit weaker than those used by Nash, however they are good enough to carry out the proof of his implicit function theorem (see $[3])$.

Lemma. Suppose $b_{1} \geqq b_{2} \geqq \cdots>0, \tau=\inf \left\{\sigma: \sum_{i=1}^{\infty} b_{i}^{\sigma}<\infty\right\}$ is finite, and $\epsilon>0$. Let $m(r)$ be the number of $b_{n}$ greater than $r^{-1}$. Then there is a $k_{0}>0$ such that for $t>\tau+\epsilon$.

$$
\sum_{j \leq m(k)} b_{j}^{-t} \leqq k^{\tau+\epsilon+t}
$$

and

$$
\sum_{j>m(k)} b_{j}^{t}<k^{\tau+\epsilon-t}(\tau+\epsilon) /(t-\tau-\epsilon), \quad \text { for } k \geqq k_{0} .
$$

Proof. From [1, p. 88] we have that $\lim \sup (\log m(k) / \log k)=\tau$. Hence for some $k_{0}>0$ it follows that $m(k)<k^{r+\epsilon}$ if $k_{0} \leqq k$. Therefore $\sum_{j \leq m(k)} b_{j}^{-t} \leqq k^{t} m(k)<k^{\tau+\epsilon+t}$.

Now, $\quad \sum_{j>m(k)} b_{j}^{t} \leqq \sum_{r=0}^{\infty}(m(k+(r+1) \Delta x)-m(k+r \Delta x)) /(k+r \Delta x)^{t}$ for $k_{0} \leqq k$ and $\Delta x>0$. Letting $\Delta x \rightarrow 0$ gives

$$
\begin{aligned}
\sum_{j>m(k)} b_{j}^{t} & \leqq \int_{k}^{\infty} x^{-t} d m(x)=\left.x^{-t} m(x)\right|_{k} ^{\infty}+\int_{k}^{\infty} t x^{-t-1} m(x) d x \\
& \leqq\left. x^{\tau+\epsilon-t}\right|_{k} ^{\infty}+\int_{k}^{\infty} t x^{\tau+\epsilon-t-1} d x=k^{\tau+\epsilon-t}(\tau+\epsilon) /(t-\tau-\epsilon) .
\end{aligned}
$$

Theorem. Suppose $\alpha \rightarrow E_{\alpha}$ is a Hilbert scale defined by $A$ and $\tau=\inf \left\{\sigma: \sum \lambda_{i}^{2 \sigma}<\infty\right\}$. Given $\epsilon>0$ there is a $K>0$, and a subsequence $S_{n}=P_{k(n)}$ such that for $\beta-\alpha>\tau+2 \epsilon$ 


$$
\left\|S_{n} u\right\|_{\beta} /\|u\|_{\alpha} \leqq n n^{(\beta-\alpha) /(\tau+\epsilon)} \quad \text { for all } u \in E_{\alpha},
$$

and

$$
\left\|\left(I-S_{n}\right) v\right\|_{\alpha} /\|v\|_{\beta} \leqq K n n^{(\alpha-\beta) /(r+\epsilon)} \quad \text { for all } v \in E_{\beta} .
$$

Proof. For $u \in E_{\alpha}$ and $\beta>\alpha$,

$$
\begin{aligned}
\left\|P_{n} u\right\|_{\beta}^{2} & =\left\|\sum_{i=1}^{n}\left(u, a_{i}\right)_{\alpha} \lambda_{j}^{2 \alpha} a_{j}\right\|_{\beta}^{2} \leqq \sum_{i=1}^{n}\left|\left(u, a_{i}\right)_{\alpha}\right|^{2} \lambda_{i}^{4 \alpha}\left\|a_{i}\right\|_{\beta}^{2} \\
& \leqq\|u\|_{\alpha}^{2} \sum_{i=1}^{n}\left\|a_{i}\right\|_{\alpha}^{2}\left\|a_{i}\right\|_{\beta}^{2} \lambda_{i}^{4 \alpha}=\|u\|_{\alpha}^{2} \sum_{i=1}^{n} \lambda_{i}^{2(\alpha-\beta)} .
\end{aligned}
$$

For $u \in E_{\beta}$ and $\beta-\alpha>\tau$ we have

$$
\begin{aligned}
\left\|\left(I-P_{n}\right) u\right\|_{\alpha}^{2} & =\left\|\sum_{k>n}\left(u, a_{k}\right)_{\beta} \lambda_{k}^{2 \beta} a_{i}\right\|^{2}=\sum_{k>n}\left|\left(u, a_{k}\right)_{\beta}\right|^{2} \lambda_{k}^{4 \beta}\left\|a_{k}\right\|_{\alpha}^{2} \\
& \leqq\|u\|_{\beta}^{2} \sum_{k>n}\left\|a_{k}\right\|_{\beta}^{2} \lambda_{k}^{4 \beta}\left\|a_{k}\right\|_{\alpha}^{2}=\|u\|_{\beta}^{2} \sum_{k>n} \lambda_{k}^{2(\beta-\alpha)} .
\end{aligned}
$$

Applying the above lemma gives that for $\beta-\alpha>\tau+\epsilon$ and $k \geqq k_{0}$ that $\left\|P_{m(k)} u\right\|_{\beta} /\|u\|_{\alpha} \leqq k^{(\tau+\epsilon+\beta-\alpha) / 2}$ for $u \in E_{\alpha}$, and $\left\|\left(I-P_{m(k)}\right) v\right\|_{\alpha} /\|v\|_{\beta}$ $\leqq k^{(\tau+\epsilon+\alpha-\beta) / 2}((\tau+\epsilon) /(\beta-\alpha-\tau-\epsilon))^{1 / 2}, v \in E_{\beta}$. Letting $K=((\tau+\epsilon) / \epsilon)^{1 / 2}$, $n=k^{(\tau+\epsilon) / 2}, \quad S_{n}=P_{m\left(n^{2 /(\tau+\epsilon)}\right)}$, and assuming $\beta-\alpha>\tau+2 \epsilon$ the result follows.

3. Commuting operators between Hilbert scales. Suppose $\alpha \rightarrow E_{\alpha}$, $\beta \rightarrow F_{\beta}$ are Hilbert scales defined by $A$ and $B$ respectively, and let $\langle,\rangle_{\beta}$ denote the inner product in $F_{\beta}$. Let $\mathcal{L}(E, F)$ be the continuous linear maps from $E$ to $F$, and define

$$
\mathfrak{L} a(E, F)=\left\{T \in \mathfrak{L}(E, F): T A^{\alpha}=B^{\alpha} T \text { for all } \alpha \in R\right\} .
$$

Proposition. Suppose $T \in \mathfrak{L}(E, F)$. Then for some $r \in R$ and all $\alpha \in R, T: E_{, \alpha} \rightarrow F,_{\alpha-r}$ is continuous. Hence $T$ has a unique extension to an element of $\mathfrak{L}\left(E_{\alpha}, F_{\alpha-r}\right)$ (also denoted by $T$ ), and the norms in the respective spaces are the same.

Proof. Since $T \in \mathfrak{L}(E, F)$ there is an $r$ such that $T: E_{, r} \rightarrow F, 0$ is continuous, and we may write $T \in \mathfrak{L}\left(E_{r}, F_{0}\right)$. For $u \in E$ and any $\alpha$, $\|T u\|_{\alpha-r}=\left\|B^{r-\alpha} T u\right\|_{0}=\left\|T A^{r-\alpha} u\right\|_{0} \leqq\|T\|\left\|A^{r-\alpha} u\right\|_{r}=\|T\|\|u\|_{\alpha}$. Hence $T: E_{, \alpha} \rightarrow F_{, \alpha-r}$ is continuous and extends to an element of $\mathfrak{L}\left(E_{\alpha}, F_{\alpha-r}\right)$ whose norm is bounded by $\|T\|$. Replacing $\alpha$ by $-\alpha$ then gives the equality of the norms.

For $T \in \mathfrak{L} a(E, F)$ we will say that ord $T \leqq r$ if $T \in \mathcal{L}\left(E_{\alpha}, F_{\alpha-r}\right)$ 
for all $\alpha \in R$. For subsets $A, B \subset E$ write $A \perp B$ iff $(a, b)_{\alpha}=0$ for all $a \in A, b \in B$, and $\alpha \in R$.

Proposition. Suppose $T \in \mathfrak{L} a(E, F)$ and $K$ denotes the kernel of $T$ in $E$. Then $E=K \oplus K^{\perp}$.

Proof. By the above Proposition $T \in \mathfrak{L}\left(E_{\alpha}, F_{\alpha-r}\right)$. Let $K_{\alpha}$ denote the kernel of $T$ in $E_{\alpha}$ and $K_{\alpha}^{\perp}$ its orthogonal complement. The result then follows from the facts that $K=\bigcap K_{\alpha}$ and $K^{\perp}=\cap K^{\perp}$.

Proposition. Suppose $T \in \mathfrak{L} a(E, F)$ is surjective. Define $L: F \rightarrow E$ by $L v=m$ iff $m \in K^{\perp}$ and $T m=v$. Then $L \in \mathcal{L} a(F, E)$ and $T L u=u$ for all $u \in F$. Moreover if ord $T \leqq r$ and ord $L \leqq s$, then $r+s \geqq 0$ and $T E_{\alpha} \supset F_{\alpha+s}$.

Proof. For the closed graph theorem it follows that $L$ is continuous. Since $L v=m, A^{\alpha} L v=A^{\alpha} m$. But $T m=v$ gives $B^{\alpha} v=B^{\alpha} T m=T A^{\alpha} m$ and since $A^{\alpha} m \in K^{\perp}, L B^{\alpha} v=A^{\alpha} m$ so that $A^{\alpha} L=L B^{\alpha}$, and $L \in \mathscr{L} a(F, E)$.

For the second part we may assume that $T$ is bijective by restricting $T$ to $K^{\perp}$. If $K^{\perp}$ is finite dimensional then ord $T=$ ord $L=0$. If $K^{\perp}$ is infinite dimensional and $r+s<0$ it follows that ord $I<0$. This would imply that for some $\epsilon>0$ and $K>0,\|u\|_{\epsilon} \leqq K\|u\|_{0}$ for all $u \in E$, which is impossible. It then follows that $T E_{\alpha} \supset F_{\alpha+\beta}$.

In the case of example (a) above $\mathcal{L} a(E, F)$ includes linear differential operators with constant coefficients, but not variable coefficients. Nonetheless since smoothing operators exist for Hilbert scales with nuclear centers a Nash type implicit function theorem can be given, and they therefore seem an appropriate setting for certain nonlinear problems.

\section{BIBLIOGRAPHY}

1. I. M. Gel'fand and N. Ja. Vilenkin, Generalized functions, Vol. 4, Academic Press, New York, 1964.

2. B. S. Mitjagin, Approximate dimension and bases in nuclear spaces, Russian Math. Surveys, London Math. Soc. 18 (1963), 59-127.

3. J. Moser, A new technique for the construction of solutions of nonlinear differential equations, Proc. Nat. Acad. Sci. U.S.A. 47 (1961) 1824-1831.

4. J. Nash, The embedding problem for Riemannian manifolds, Ann. of Math. 63 (1956), 20-63.

5. R. Palais, Seminar on the Atiyah-Singer index theorem, Annals of Mathematics Studies No. 57, Princeton Univ. Press, Princeton, N. J., 1965.

6. J. Schwartz, Nonlinear functional analysis, Mimeographed notes, New York Univ., New York, 1964.

NORTHEASTERN UNIVERSITY 